\title{
Desarrollo de competencias digitales de estudiantes universitarios en contextos informales de aprendizaje
}

\section{Development of digital skills of university students in informal learning contexts}

\author{
Karen S. LÓpez-GIL ${ }^{1 *}$ \\ karenlopez@javerianacali.edu.co, https://orcid.org/0000-0001-9826-0799 \\ María Luisa Sevillano García** \\ mlsevillano@edu.uned.es,https://orcid.org/0000-0002-2841-8738 \\ * Pontificia Universidad Javeriana, Colombia \\ **Universidad Nacional de Educación a Distancia, UNED, España
}

\section{Resumen:}

Este artículo busca evidenciar la percepción de estudiantes universitarios sobre el desarrollo de competencias digitales por fuera de los contextos educativos formales. Para ello, se llevó a cabo una investigación mixta secuencial con ingresantes universitarios de una institución privada de la ciudad de Cali, Colombia. Como técnicas de recolección de información se implementaron un cuestionario electrónico y dos grupos de discusión. Entre los principales resultados se encontró que los estudiantes tienen una percepción heterogénea respecto al desarrollo de las distintas áreas de la competencia digital, siendo más fuertes las relacionadas con la interacción y la creación/ edición de contenidos gráficos. Las de menor desarrollo son las competencias de seguridad y resolución de problemas. Los estudiantes dan cuenta de un amplio número de prácticas digitales que han aprendido de forma autónoma, a través de la interacción con otros sujetos, con

\begin{abstract}
:
The purpose of this article is to explore the perception of university students about the development of digital skills in informal educational contexts. To this end, a sequential mixed research was conducted with university students from a private institution in the city of Cali, Colombia. The data collection techniques consisted of an electronic questionnaire and two discussion groups. Among the main results, it was found that students have a heterogeneous perception regarding the development of the different areas of digital skills, being stronger those related to the interaction and the creation/ edition of graphic content. The lowest values were reported for security competencies and problem solving. The students referred a wide number of digital practices that they had learned autonomously, through interaction with other individuals, with web resources and from trial-error personal experiences. However, these competences are not usually transferred to the academic
\end{abstract}

1 Dirección para correspondencia (correspondence address):

Karen S. López Gil. Calle 18 \# 118 - 250, edificio Samán, oficina 3.05 Cali (Colombia) 
Desarrollo de competencias digitales de estudiantes universitarios en contextos informales de aprendizaje

Karen S. López-Gil y María luisa Sevillano García

recursos propios de la web y a partir de experiencias personales de ensayo y error, pero estas competencias no suelen transferirse al ámbito académico. Finalmente, se presentan algunas propuestas que pueden plantearse como ejes articuladores del desarrollo de competencias digitales dentro y fuera del aula.

\section{Palabras clave:}

Competencia digital; aprendizaje informal; dispositivos móviles; universidad. field. Finally, some proposals are presented. They can be considered as articulating axes for the development of digital competences inside and outside the classroom.

\section{Key Words:}

Digital skills; informal learning; mobile devices; higher education.

\section{Résumé:}

Cet article vise à montrer la perception des étudiants sur le développement des compétences numériques en dehors des contextes éducatifs formels. Pour ce faire, une recherche mixte séquentielle avec des étudiants universitaires inscrits dans une institution privée de la ville de Cali, en Colombie, a été menée. Comme techniques de collecte d'informations, un questionnaire électronique et deux groupes de discussion ont été mis en place. Parmi les principaux résultats, nous avons constaté que les étudiants ont une perception hétérogène du développement des différents domaines de compétence numérique, les relations avec l'interaction et la création / édition de contenu graphique étant plus fortes. Les compétences les moins développées sont celles de la sécurité et de la résolution de problèmes. Les élèves rendent compte $d^{\prime} u n$ large éventail de pratiques numériques qu'ils ont appris de façon autonome, grâce à l'interaction avec d'autres sujets, avec les ressources du web et des expériences personnelles d'essais et d'erreurs, mais ces compétences ne sont généralement pas transférés au domaine académique. Enfin, nous présentons quelques propositions qui pourraient être envisagées comme I'articulation des axes de développement des compétences numériques à l'intérieur et à l'extérieur de la salle de class.

\section{Mots-clés:}

Compétence numérique; apprentissage informel; appareils mobiles; université.

Fecha de recepción: 21-8-2018

Fecha de aceptación: 9-1-2019

\section{Introducción}

En las primeras dos décadas del siglo XXI, la humanidad ha experimentado cambios vertiginosos respecto a las formas de acceder, comunicar y almacenar la información. El acceso casi ilimitado a múltiples fuentes demanda nuevas competencias por parte de los sujetos que aprenden. Si bien las tecnologías por sí mismas no implican cambios en los modos de aprender, sí favorecen la aparición de prácticas, de nuevos alfabetismos, de formas de relacionarse en contextos sociales específicos que están mediados por artefactos (Lankshear y Knobel, 2011). Tal es el caso de 
los dispositivos móviles, que han difuminado los límites temporales y espaciales del acceso a la información.

A pesar de estas transformaciones, no podemos referirnos a los aprendices del nuevo milenio como un conjunto homogéneo de sujetos, con habilidades y competencias que vienen predeterminadas por aspectos generacionales. Las relaciones entre los jóvenes y las tecnologías son heterogéneas, diversas, y están enmarcadas en experiencias de distintos contextos.

En The NMC Horizon Report: 2017 Higher Education Edition, que proyecta las tendencias en educación superior en el mundo, se plantea como desafío urgente la mejora en la alfabetización digital de estudiantes universitarios. Se plantea trascender el manejo operativo de las tecnologías actuales y propone a un uso consciente, razonado y crítico de estas en contextos específicos (Instituto Nacional de Tecnologías Educativas y de Formación del Profesorado [INTEF], 2017a). Este informe también identifica como desafío a corto plazo el establecimiento de puentes entre prácticas de aprendizaje en contextos formales y contextos informales.

El interés por estudiar los contextos informales de aprendizaje no es nuevo. Ya desde los años sesenta, desde la Psicología y la Antropología se adelantaban investigaciones relacionadas con los aprendizajes fuera de la escuela y se le daba valor a este campo como objeto de estudio. Esta tradición ha continuado en distintas disciplinas (Paradise, 2005). Además, como coinciden González (2009) y Cassany (2013), con la masificación de Internet estas prácticas se han reconfigurado y el aprendizaje informal se ha potenciado, pues la red ha favorecido la descentralización de las instituciones, el acceso a la información y la conformación de comunidades al margen de las entidades formales.

En la actualidad, Ilaman la atención los caminos que recorren los estudiantes para desarrollar sus competencias digitales, debido a que el uso cercano de las tecnologías puede volver invisibles estos aprendizajes. Al respecto, Cobo y Moravec (2011) plantean el concepto "aprendizaje invisible" para dar cuenta de aquello que las personas construyen sin que sea regulado por el contexto formal, de hecho, muchas veces sin que sea validado, pero que hace parte de los desarrollos de cada sujeto y que incide en sus desempeños tanto en el ámbito académico como en el laboral.

A esto sumamos el importante papel que tienen tanto las competencias digitales como las posibilidades de gestionar los propios aprendiza- 
jes, pues ambos aportan al "Lifelong Learning", necesario para continuar aprendiendo en diferentes momentos del ciclo vital, aprovechando al máximo lo que se construye en contextos formales, pero también en el ámbito laboral y de ocio (Unesco, 2010).

A partir de estos planteamientos, este artículo busca identificar las percepciones de estudiantes universitarios respecto al desarrollo de competencias digitales al margen del contexto académico y plantear algunos ejes que permitan articular este desarrollo dentro y fuera del aula.

\section{Marco teórico}

Esta investigación se situó desde un enfoque sociocultural y ecológico de la tecnología educativa (Domínguez y Trillo Miravalles, 2014). Desde este enfoque, si bien se reconoce el papel de las tecnologías de la comunicación y de la información en las prácticas de los sujetos, no se asume un determinismo tecnológico, al considerarse que las prácticas se configuran en contextos sociales, a través de la interacción con otros y con artefactos propios de la cultura. Desde este enfoque, se concibe que los estudiantes construyen conocimientos tecnológicos en distintos ámbitos y que es necesario favorecer su conexión y validación.

En este marco, la competencia digital juega un rol fundamental, pues además de ser identificada como una de las ocho competencias clave para el aprendizaje permanente (Comisión Europea, 2007), es aquella que permite una utilización óptima de los recursos tecnológicos. Por su parte, los contextos de aprendizaje informal aportan al desarrollo de esta competencia a través de las múltiples posibilidades de interacción con contenidos, personas y herramientas.

\subsection{Competencia digital}

En Educación para la ciudadanía mundial, la Unesco (2016) destaca el uso de las tecnologías de la información y de los medios de comunicación como uno de los requisitos esenciales para la formación académica y para la participación social. En un entorno marcado por el desarrollo tecnológico, es necesario que los individuos construyan competencias que les permitan desenvolverse de forma eficiente, reflexiva y crítica. 
Aunque el concepto de competencia digital ha sido ampliamente discutido y contrastado con otras nociones cercanas como competencias informacionales, tecnológicas y mediáticas (Dornaleteche-Ruiz, Buitrago-Alonso y Moreno-Cardenal, 2015), asumimos aquí la definición del Parlamento Europeo y el Consejo de la Unión Europea (2006), que concibe la competencia digital como "...el uso seguro y crítico de las Tecnologías de la Sociedad de la Información (TSI) para el trabajo, el tiempo libre y la comunicación. Se sustenta en las competencias básicas en materia de TIC: uso de ordenadores para obtener, evaluar, almacenar, producir, presentar e intercambiar información, y para comunicar y participar en redes de colaboración a través de Internet". Tendríamos que sumar a esta definición el uso de dispositivos móviles, que han ganado protagonismo en los últimos años.

La competencia digital no se refiere a un concepto unidimensional y estático, sino que implica la articulación de múltiples variables. Instituciones como la International Society for Tecnology in Education (ISTE) han establecido una serie de estándares que permiten evidenciar los distintos componentes de la competencia digital, entre los que se incluyen: creatividad e innovación; comunicación y colaboración; investigación y manejo de información; pensamiento crítico, solución de problemas y toma de decisiones; ciudadanía digital; y conceptos de las TIC (Gutiérrez, Cabero y Estrada, 2017).

Por su parte, el Instituto Nacional de Tecnologías Educativas y de Formación del Profesorado (INTEF, 2017b) ha construido un marco de competencia digital que incluye cinco grandes áreas y tres niveles de desempeño (avanzado, intermedio y básico). Aunque este marco está dirigido especialmente a docentes, su grado de detalle y organización permite su adaptación a otros contextos y poblaciones. Las áreas planteadas por INTEF son el uso de la información, la comunicación y colaboración, la creación de contenidos digitales, la seguridad y la resolución de problemas.

\subsection{Aprendizaje informal mediado por tecnologías}

El aprendizaje informal suele ser definido por oposición al aprendizaje formal, es decir, como aquel que tiene lugar fuera de la escuela o, más específicamente, del currículo (Cross, 2006). Aunque no hay consenso entre los autores respecto a su definición, sí hay coincidencias respecto 
Desarrollo de competencias digitales de estudiantes universitarios en contextos informales de aprendizaje

Karen S. López-Gil y María luisa Sevillano García

a su importancia, por considerarse como valioso por sí mismo y, en ningún caso, como inferior al formal (Infed, 2010).

Como plantean Vázquez-Cano, López y Sánchez-Serrano (2013):

\begin{abstract}
En los años recientes han aparecido infinitas posibilidades para la formación autodirigida, los encuentros entre docente y alumno en contextos online, o el aprendizaje entre pares, por poner algunos ejemplos. Todas estas instancias, sin embargo, se han quedado hasta ahora casi siempre fuera de lo que se considera educación seria, formal... Estas habilidades más informales tienen un peso enorme en la nueva economía y en la nueva sociedad (p. 87).
\end{abstract}

Las formas de construir los aprendizajes en contextos informales son variadas y pueden ser intencionales o incidentales (Sackey, Nguyen y Grabill, 2015). Ejemplos de aprendizaje informal intencional son la lectura o la búsqueda de asesoría, mientras que aprendizajes informales inesperados podrían relacionarse con el juego, la exploración y la interacción en comunidades. De acuerdo con Paradise (2005), una de las principales características del aprendizaje informal es la motivación, pues suele centrarse en los intereses particulares de los sujetos o grupos. La fuerte motivación lleva a la autogestión del aprendizaje, cuando se tiene un propósito definido, o a la apropiación tácita del conocimiento, cuando es producto de interacciones espontáneas.

Si bien siempre han existido distintos contextos en los que los sujetos pueden aprender por fuera de la escuela, las tecnologías, sobre todo Internet, han potenciado el aprendizaje informal. Dos escenarios han marcado fuertemente estos cambios en los últimos años. El primero es el acceso a los recursos que facilitan el aprendizaje autogestionado. Los recursos educativos digitales abiertos y los MOOC son dos ejemplos de herramientas que facilitan la construcción de itinerarios de aprendizaje propios. El segundo escenario se relaciona con las posibilidades de comunicación y colaboración que dan lugar a comunidades. Desde hace dos décadas, Wenger (2000) planteó el concepto de "comunidades de práctica", que se refiere a la agrupación de personas que comparten unas finalidades, que interactúan frecuentemente entre sí y que desarroIlan prácticas de comunicación particulares, que permiten incluso la generación de una identidad colectiva. Posteriormente, otros autores han replanteado la noción de comunidad de práctica, como es del caso de Gee (2017) que plantea 'espacios de afinidad' como un concepto más 
cercano a lo que sucede en los entornos digitales. En los espacios de afinidad hay un propósito compartido, se dan formas de participación muy diversas, se generan recursos propios, se construyen identidades y se establecen roles que permiten el funcionamiento y la continuidad del grupo. Ejemplos de espacios de afinidad sería los grupos de scanlation (escaneado y traducción de cómics), que suelen tener unas complejas formas de organización que les permiten lograr su propósito.

La articulación entre el aprendizaje formal y el informal puede dar lugar a un aprendizaje mucho más potente e integrado, denominado por algunos autores como aprendizaje expandido, extendido, aumentado o invisible (Cobo y Moravec, 2011). En gran parte, estos aprendizajes se ven favorecidos por la mediación de los dispositivos electrónicos.

\section{Marco empírico}

\subsection{Objetivos}

Esta investigación buscaba evidenciar la percepción de estudiantes universitarios sobre el desarrollo de sus competencias digitales por fuera de contextos educativos formales. Para ello, se plantearon objetivos específicos que pretendían caracterizar la autopercepción de la competencia digital de acuerdo con las cinco áreas propuestas por INTEF (2017b), la generación de un inventario de aprendizajes digitales informales, la identificación de estrategias de autoformación en competencia digital, el reconocimiento de herramientas y recursos para el aprendizaje informal, y las posibilidades de transferencia de las competencias digitales al contexto académico.

\subsection{Enfoque}

La investigación se planteó desde un enfoque mixto, el cual integra propósitos, métodos y técnicas de recolección y de análisis de información tanto cuantitativas como cualitativas (Hernández, Fernández y Baptista, 2014). El diseño fue secuencial con una primera fase cuantitativa que consistió en identificar, mediante un cuestionario, las percepciones de los estudiantes respecto al desarrollo de la competencia digital en contextos informales de aprendizaje, y una segunda fase cualitativa en la 
que los resultados se ampliaron a través de la realización de dos grupos de discusión.

\subsection{Contexto y población}

La investigación se desarrolló en una institución de educación superior en la ciudad de Cali, Colombia, en el marco de una asignatura introductoria de lengua en modalidad semipresencial, en la que se hace un uso importante de las competencias digitales.

La población estuvo constituida por 212 ingresantes de seis carreras de una facultad de ingenierías. $72 \%$ de los participantes fueron hombres y $28 \%$ mujeres. Su rango de edad estuvo entre los 16 y los 19 años y se encontraban cursando primer semestre.

\subsection{Técnicas de recolección de información}

En la primera fase, se aplicó un cuestionario. Este instrumento incluyó preguntas sobre el uso de dispositivos electrónicos, la autopercepción de la competencia digital en cada una de las áreas planteadas por INTEF, las competencias que los estudiantes consideran que han desarrollado en contextos informales, las estrategias de formación en competencia digital, los recursos y herramientas que usan, así como las posibilidades de articulación del uso de competencias entre los contextos formales y no formales. El cuestionario estuvo compuesto por 17 preguntas de opción múltiple y 3 preguntas abiertas (ver anexo 1) y se aplicó en línea.

Posterior al análisis de los resultados del cuestionario, se llevaron a cabo dos grupos de discusión con un total de 17 estudiantes. El grupo de discusión se considera una técnica útil en el estudio de percepciones, concepciones, actitudes, sentimientos y prácticas de los sujetos respecto a un tema (Gil Flores, 1992). En los grupos realizados se profundizó en los resultados del cuestionario, particularmente en las formas como se desarrolla la competencia digital en contextos informales y las ventajas y desventajas de transferir estas competencias al ámbito académico.

Para analizar los datos de naturaleza cuantitativa se presentan a continuación gráficos de frecuencia. Por otra parte, la información cualitativa se trabajó mediante el análisis de contenido, en el que se analizaron los mensajes de los participantes atendiendo al contexto de producción e identificando temáticas destacadas y recurrentes (Ballesteros y Mata, 
2014). Los participantes se identifican con la letra E y con números del 1 al 17.

\section{Resultados}

\subsection{Uso de dispositivos electrónicos}

A partir de la encuesta, se identificó que los participantes cuentan, en su mayoría, con tres dispositivos electrónicos. $85 \%$ cuentan con teléfono inteligente, $80 \%$ con ordenador de escritorio, $76 \%$ con ordenador portátil, $50 \%$ con tableta o Ipad y $8 \%$ con e-readers. Todos tienen acceso a internet, a través de redes públicas $(100 \%)$, redes privadas $(81 \%)$, plan de datos (55\%) o internet móvil (9\%).

Los dispositivos electrónicos se usan con múltiples propósitos, principalmente para actividades de comunicación (96\%), entretenimiento (92\%), acceso a información en la web (75\%), aprendizaje (68\%) y otros (9\%).

$\mathrm{Al}$ indagar por los lugares de uso de los dispositivos, se identifica que más de la mitad de los estudiantes utiliza los equipos tanto en espacios de estudio de la universidad como en el hogar (figura 1).

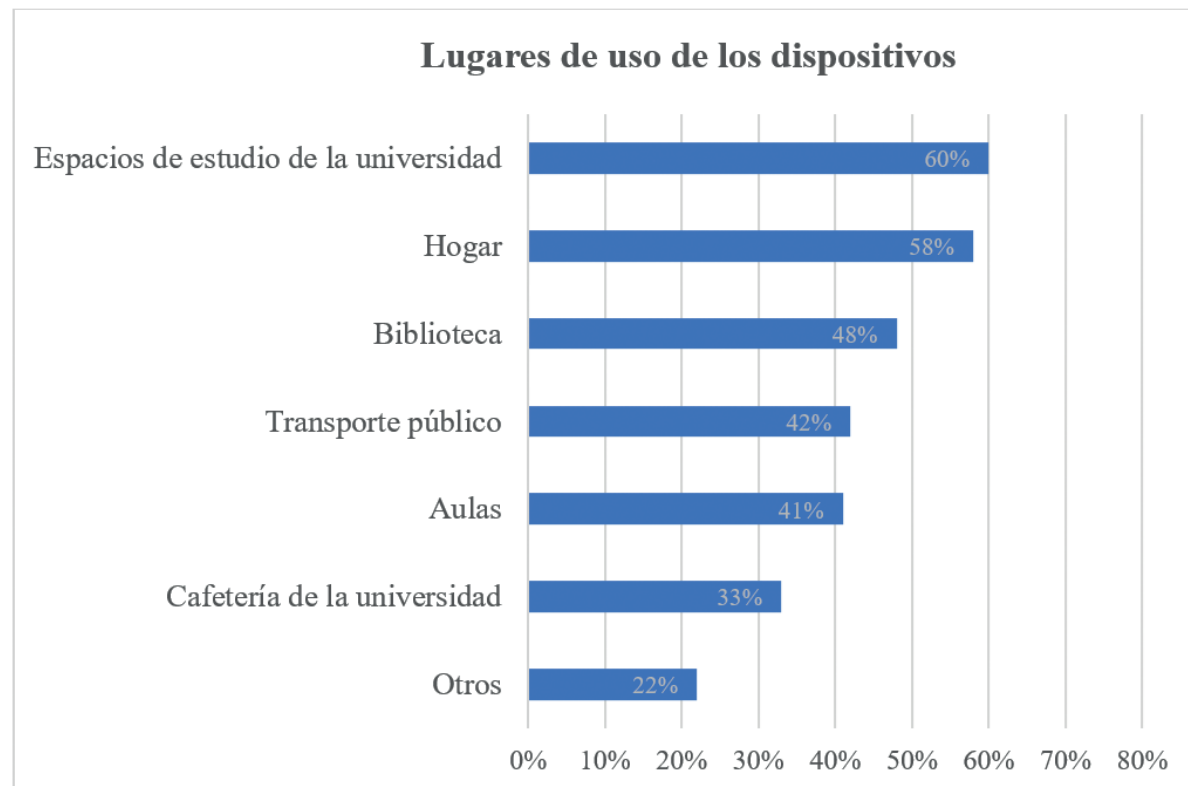

Figura 1. Lugares de uso de los dispositivos electrónicos. 


\subsection{Autopercepción de la competencia digital}

Esta categoría se refiere a la valoración que los estudiantes hacen sobre el desarrollo de sus competencias digitales en tres niveles de desempeño: básico, intermedio y avanzado. Para determinar las áreas o dimensiones de la competencia digital, se retomó la taxonomía propuesta por INTEF (2017b), que incluye las subcompetencias de información, comunicación y colaboración, creación de contenidos, seguridad, y resolución de problemas.

\subsection{1. ÁREA 1}

El área de información y alfabetización informacional se refiere a la capacidad de las personas para trabajar con los contenidos de la web, desde el proceso de navegación, búsqueda y filtro de las fuentes, hasta su análisis, evaluación y posterior almacenamiento y recuperación.

En esta primera área, los ingresantes universitarios se autoperciben, en general, con un nivel avanzado en los procesos de navegación, búsqueda y filtro de información, así como en su almacenamiento y recuperación (figura 2).

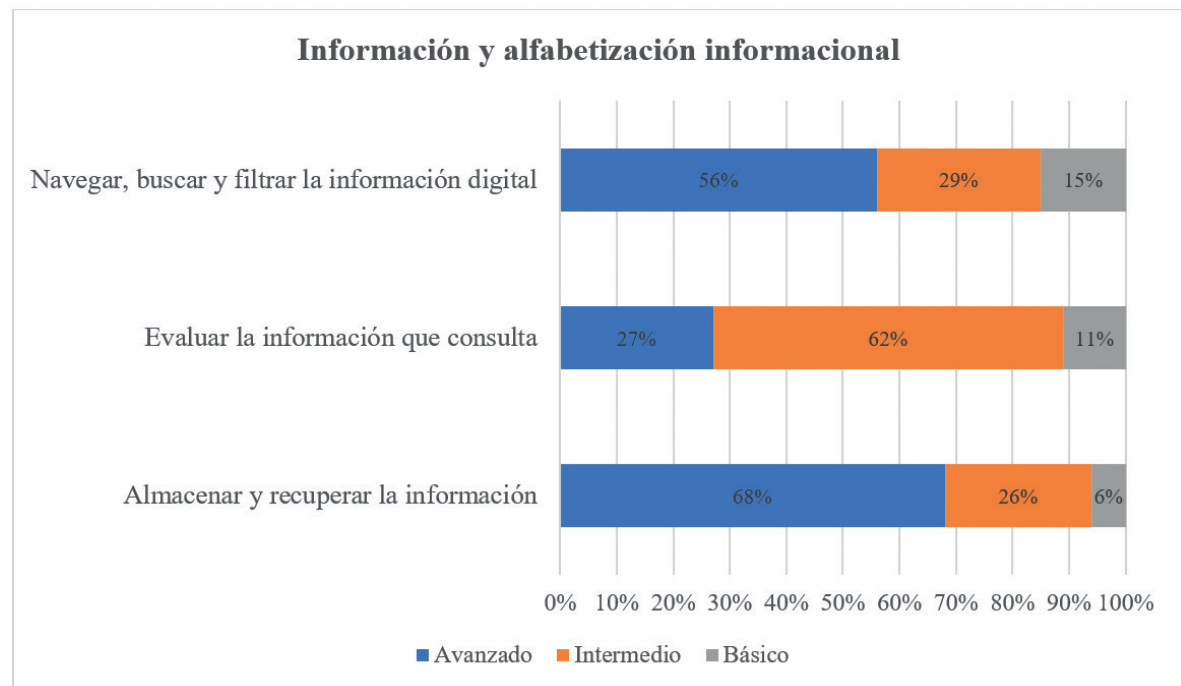

Figura 2. Autopercepción de la competencia de información y alfabetización informacional. 
Llama la atención que la evaluación de esta información sea considerada como una capacidad de nivel intermedio, pues los otros procesos pierden efectividad cuando no se logra una evaluación adecuada de las fuentes.

En el grupo de discusión, por su parte, los estudiantes confirman que los procesos de búsqueda y de almacenamiento son más sencillos que los de evaluación de las fuentes:

E2: "Si tienes las palabras adecuadas Google te muestra miles de resultados, incluso los más parecido a lo que quieres, también es muy fácil guardar todo, sea en la misma nube o al mandar por correo o incluso en una memoria USB". E4: "Es fácil buscar, pero lo que no es fácil es saber si la información es adecuada, pues se encuentra mucha información perjudicial en internet, noticias falsas, artículos con datos incorrectos, etc.".

\subsection{2. ÁREA 2}

El área de comunicación y colaboración se refiere a interactuar adecuadamente en entornos digitales, usar las herramientas en línea para compartir información y participar de comunidades y redes, sean académicas o informales.

De acuerdo con la figura 3, en los componentes relacionados con la interacción y con compartir información en entornos digitales, se evidencia una autopercepción muy positiva de los estudiantes, quienes se sitúan en niveles avanzados.

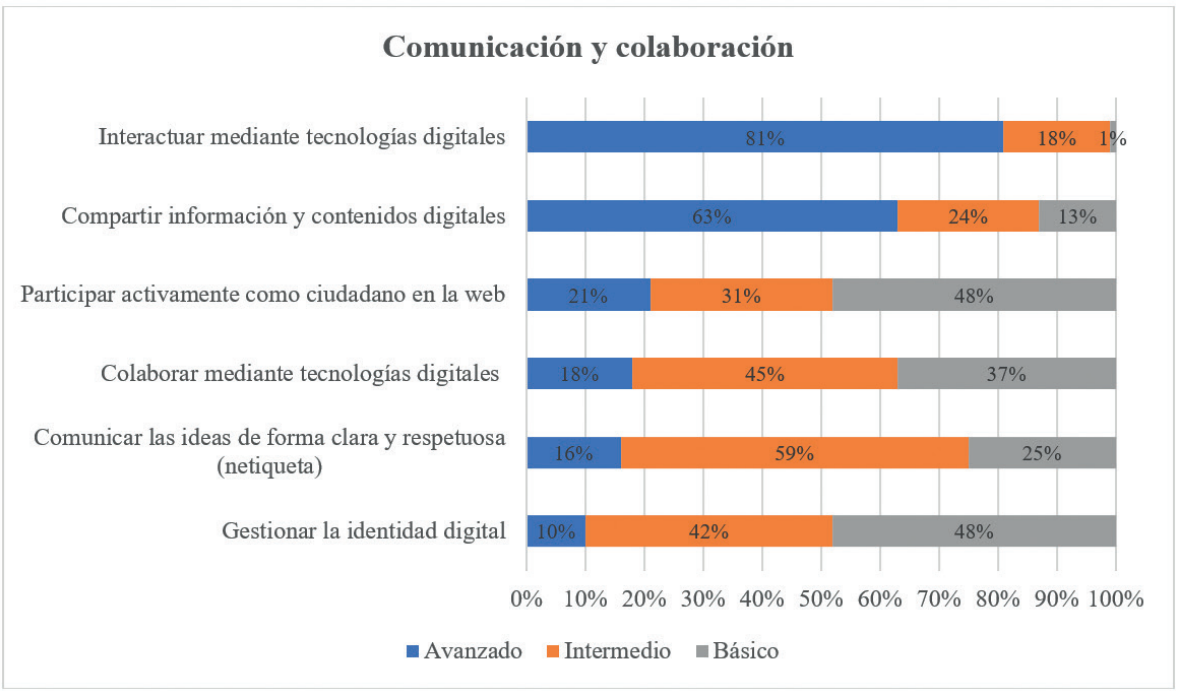

Figura 3. Autopercepción de la competencia de comunicación y colaboración. 
Esto contrasta con los otros elementos de la competencia, ya que se evalúan en niveles básicos en la participación ciudadana, la colaboración con otros y en la gestión de su identidad digital. En un nivel medio aparece la netiqueta o competencia para comunicarse de forma respetuosa en la red, siguiendo las reglas de cortesía propias de entorno digital. En el grupo de discusión, los jóvenes destacan la ausencia de reflexiones respecto al uso de las tecnologías para la participación social, así como sus inquietudes sobre cómo los ven los demás en entornos digitales:

E15: "... por ejemplo, lo de usar las tecnologías para participar como ciudadano no lo había considerado nunca como una habilidad que se relaciona con lo digital".

E17: "... uno poco se pone a pensar cómo es que pueden vernos los demás de acuerdo con lo que publicamos en la red, si piensan que alguno es 'vago' por publicar muchas fotos de salidas o si otro es juicioso porque todo lo que publica es sobre parciales y trabajos".

\subsection{3. ÁREA 3}

El área de creación de contenidos se refiere a la creación, edición y reelaboración de contenidos digitales en distintos modos de representación (texto, imagen, sonido, multimodal), respetando los derechos de autor y las licencias de uso y reproducción.

Como se evidencia en la figura 4, los participantes se autoperciben con un mayor dominio en la creación y reelaboración o edición de contenidos digitales. Se identifica una percepción de nivel intermedio en el respeto de los derechos de autor o de reconocimiento de las licencias de uso. El criterio relacionado con la programación es en el que hay una percepción menos favorable, ya que más del $70 \%$ se conciben como usuarios básicos. 


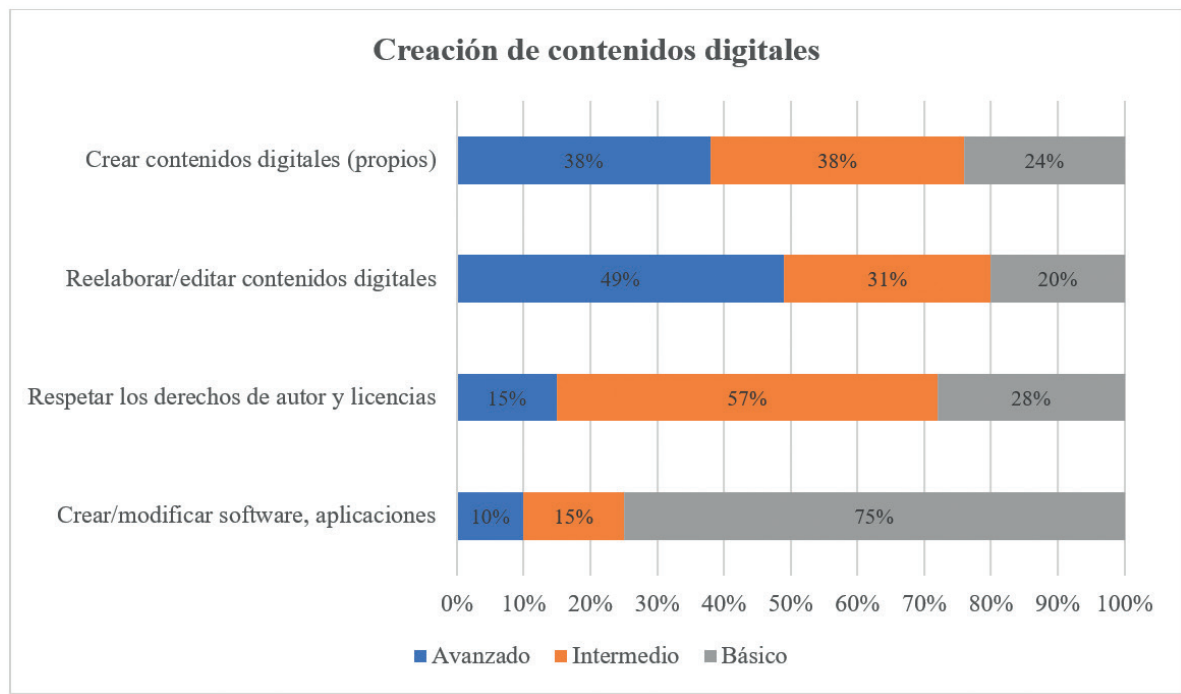

Figura 4. Autopercepción de la competencia de creación de contenidos digitales.

En el grupo de discusión, los estudiantes destacan que la creación y edición de contenido se relaciona principalmente con imágenes estáticas (retoques de fotografías y elaboración de memes) y que encuentran diferencias importantes en el uso de información en contextos informales y formales:

E7: "A mí no me parece tan claro lo del plagio. En internet todo parece ser público, se puede usar lo que otros han hecho, pero una vez llegas a la universidad hay mucho problema con esto, no puedes solo copiar y pegar. Creo que es lo que más nos complica".

E11: "En mi caso lo que más hago es desarrollar o modificar memes, retocar fotografías en Photoshop o con las mismas herramientas del Smartphone".

\subsection{4. ÁREA 4}

El área de seguridad se refiere a cómo los usuarios protegen información y datos personales en contextos digitales, así como el uso responsable que hacen de las tecnologías para el cuidado de la salud y del ambiente.

En la figura 5 se evidencia una distribución equilibrada de los estudiantes en cada uno de los niveles. Los estudiantes se autoperciben con mayores niveles de competencia en aspectos como la protección de los dispositivos y los contenidos. 
Desarrollo de competencias digitales de estudiantes universitarios en contextos informales de aprendizaje

Karen S. López-Gil y María Luisa Sevillano García

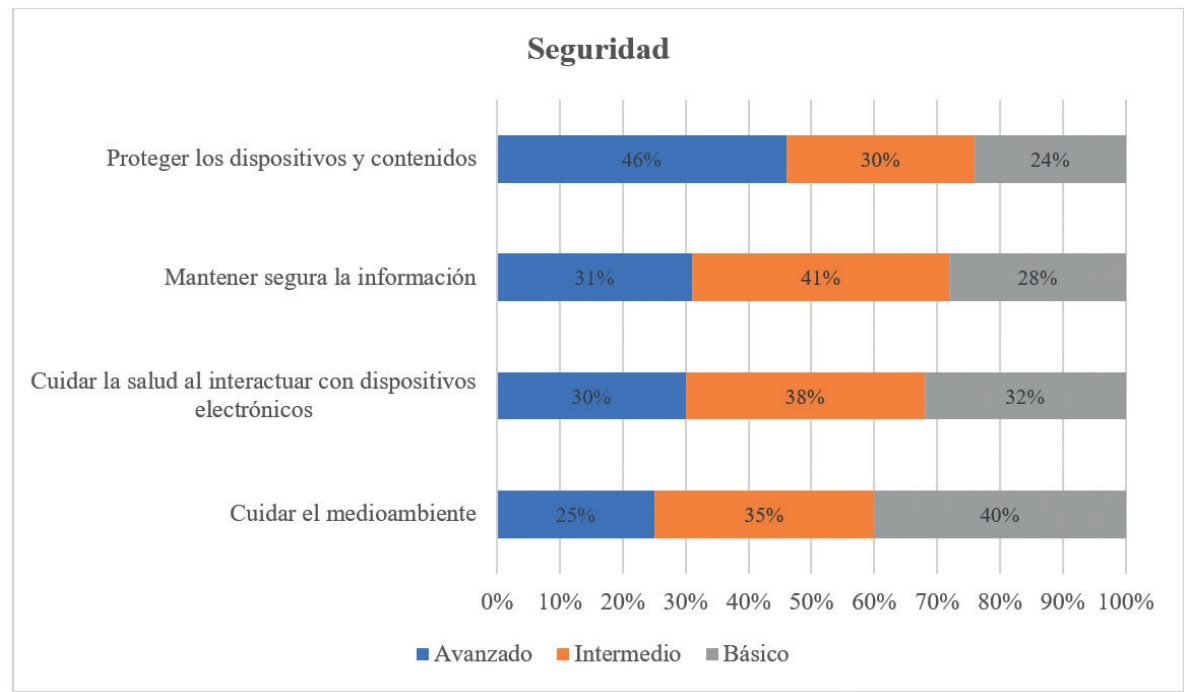

Figura 5. Autopercepción de la competencia de seguridad.

En el grupo de discusión, se planteó este cuidado a través del uso de programas antivirus y también se destacaron algunas reflexiones sobre el cuidado de la salud y del entorno:

E2: "Siempre es necesario mantener el antivirus en los equipos, mucha gente no lo usa en el celular, pero allí también es necesario...también debemos cuidarnos nosotros de la luz, la radiación...".

E7: "Otro punto creo que es la obsolescencia ya que las industrias siempre quieren vender más y más y nos muestran modelos nuevos... entonces entiendo que cuidarnos y cuidar el ambiente se relaciona con esto, con controlar las compras, con no volverse adicto ni generar exceso de basura electrónica".

\subsection{5. ÁREA 5}

El área de resolución de problemas se relaciona con la capacidad del usuario para identificar sus necesidades en los entornos digitales, evaluar alternativas y tomar decisiones razonadas.

De acuerdo con la figura 6, la mayoría de los participantes se autopercibe en un nivel avanzado para resolver problemas con las herramientas básicas, al considerarlas de manejo cotidiano. No obstante, con herramientas más avanzadas o con el uso creativo e innovador de la tecnología, esta autovaloración disminuye. 


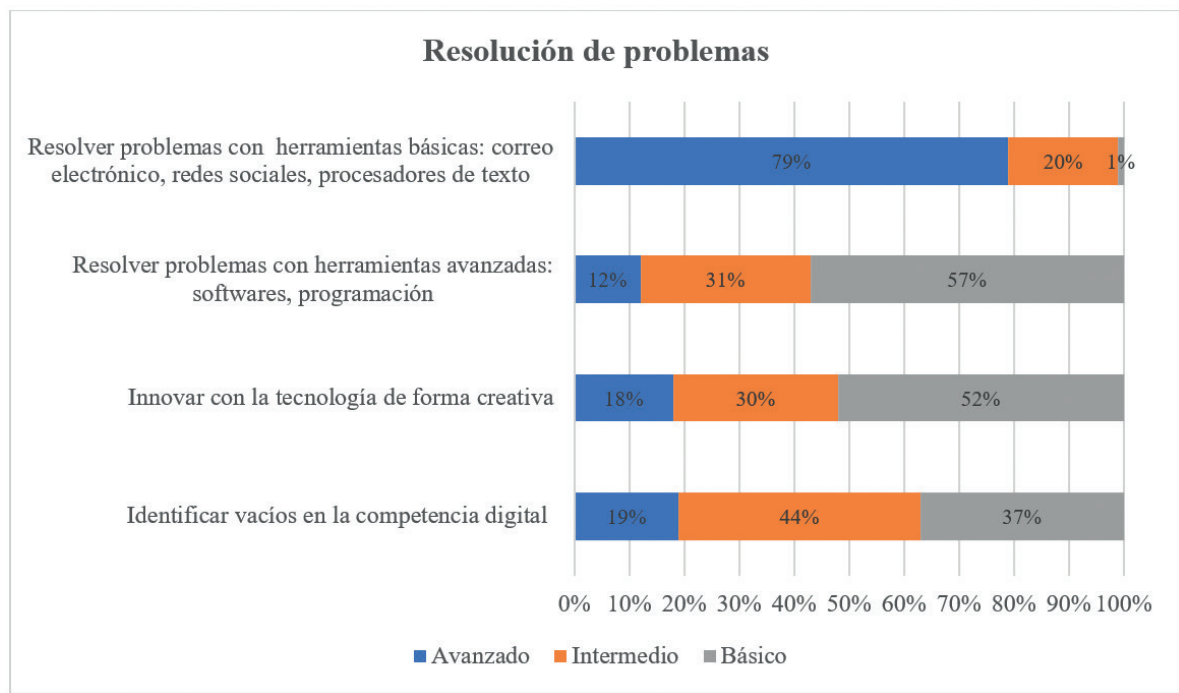

Figura 6. Autopercepción de la competencia de resolución de problemas.

En el grupo de discusión, los estudiantes indican que las expectativas de la sociedad respecto al dominio tecnológico de las nuevas generaciones suelen ser altas:

E15: "Yo me considero un usuario normal, no tan avanzado como se espera porque somos nativos digitales...".

E17: "Sí nacimos con la tecnología rondando y la usamos mucho, pero las opciones son muy amplias y nosotros no podemos saberlo todo. Es más la expectativa que se tiene sobre nosotros que lo que realmente podemos y sabemos hacer".

\subsection{Inventario de aprendizajes digitales informales}

Esta categoría buscaba indagar por los desarrollos de la competencia digital que los estudiantes han logrado en contextos informales de aprendizaje.

Para ello, en primer lugar, se hizo una pregunta de opción múltiple en la que los estudiantes podían escoger varias opciones de aprendizajes. Posteriormente, se hizo una pregunta abierta a modo de inventario para reconocer aspectos no contemplados en la pregunta cerrada.

En la figura 7, se evidencia que el manejo de las redes sociales y la 
Desarrollo de competencias digitales de estudiantes universitarios en contextos informales de aprendizaje

Karen S. López-Gil y María luisa SeVillano García

edición de imágenes son los principales aprendizajes logrados de forma autogestionada. Aparece también el manejo de software básico y en menor medida creación de otros tipos de contenido y programación.

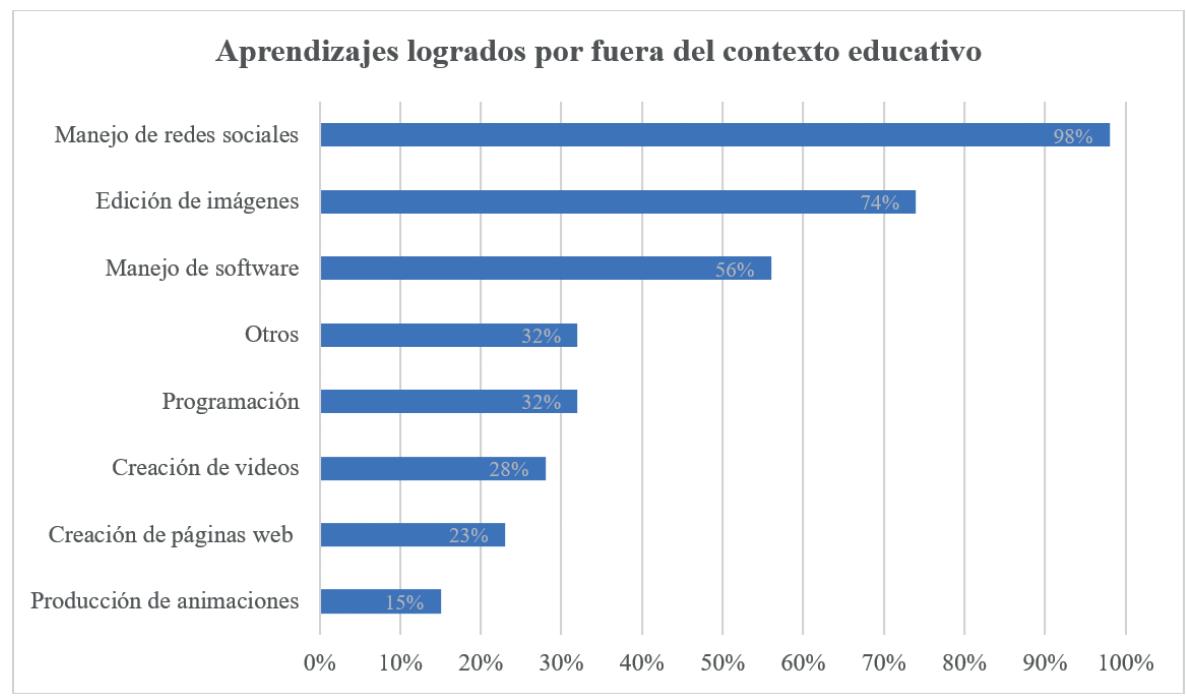

Figura 7. Aprendizajes logrados por fuera del contexto educativo.

En la pregunta abierta, se solicitó a los estudiantes que hicieran un listado - con mínimo 3, máximo 10 opciones- con habilidades del entorno digital que hubiesen aprendido autónomamente. Se obtuvieron 1136 respuestas y se hizo un análisis de contenido cuantitativo con el software Atlas Ti 8.0, evidenciado en la tabla 1. Se identificó la frecuencia de aparición de cada habilidad, se integraron las que apuntaban al mismo significado y luego se clasificaron de acuerdo con las cinco áreas de la competencia digital planteadas por INTEF (2017b). 
Tabla 1

Inventario de aprendizajes informales

\section{Creación de contenidos digitales Comunicación y colaboración}

372 respuestas agrupadas en 13 sub- 287 respuestas agrupadas en 11 subcategorías: categorías:

1. Edición de imágenes (93)

2. Curación de contenidos (56)

3. Creación de imágenes/memes (48)

4. Programación (32)

5. Creación de contenido literario (26)

6. Creación de contenido informativo (23)

7. Creación de videos y videotutoriales (32)

1. Manejo de redes sociales (92)

2. Comunidades de videojuegos (41)

3. Expresión de la opinión política (41)

4. Contribución a causas sociales (26)

5. Documentos compartidos en línea (25)

6. Pagos en línea (18)

7. Venta de productos (16)

8. Creación de animaciones (21)

9. Creación de páginas web (21)

8. Reclamos y denuncias en línea (13)

9. Recolección de firmas (7)

10. Creación de esquemas u organiza- 10.Creación de comunidades o grudores, mapas, nubes (9) pos (5)

11. Publicación de contenido en IS- 11. Cuidado de la imagen digital (3) SUU, Slideshare, Academia (6)

12.Scanlation (3)
Información y alfabeti- Seguridad zación digital

149 respuestas agrupa- mas

213 respuestas agrupa- das en 7 subcategorías: 115 respuestas agrupadas en 8 subcategorías: 1. Bloqueo de usuarios das en 6 subcategorías:

1. Navegación por inter- (36) 1. Compresión de donet (79)

2. Protección de conte-

2. Almacenamiento en nidos (28) la nube (56)

3. Ajustes de privacidad (26)

4. Instalación y uso de 3. Cambio de formato de ción (23)

4. Filtro de datos (15) antivirus (24) cumentos para envío (28)

3. Búsqueda de informa-

5. Etiquetado y organiza- 5. Comprobación de dación de archivos en la nube (14)

6. Uso de diccionarios (14)

7. Uso de traductores (6)

8. Búsqueda de palabras al interior del texto o página web (6) tos en internet (19) 5. Resolución de proble-

6. Bloqueo de contenido indeseado (9) mas con computador o impresora (13)

7. Denuncia de conteni- 6 . Conseguir espacio en $\operatorname{dos}(7)$ la nube (12) 
Este inventario abierto coincide con los resultados de otras preguntas del cuestionario, así como de otras investigaciones, en donde se identifica que los jóvenes tienen mayores fortalezas en las áreas de creación de contenidos básicos (principalmente gráficos) y de comunicación y colaboración. No obstante, los resultados permiten evidenciar con un mayor grado de detalle aquellas prácticas que los estudiantes dominan y que pueden potenciarse en el aula.

\subsection{Estrategias de formación competencia digital}

Esta categoría indaga por los contextos y estrategias de formación que los jóvenes usan, de forma intencional, para atender a sus necesidades en el medio digital. La figura 8 evidencia como principal estrategia la consulta de información en internet, tanto en formato textual como a través de videotutoriales. Aparecen con menor frecuencia la participación en comunidades de práctica y la formación específica a través de cursos en línea.

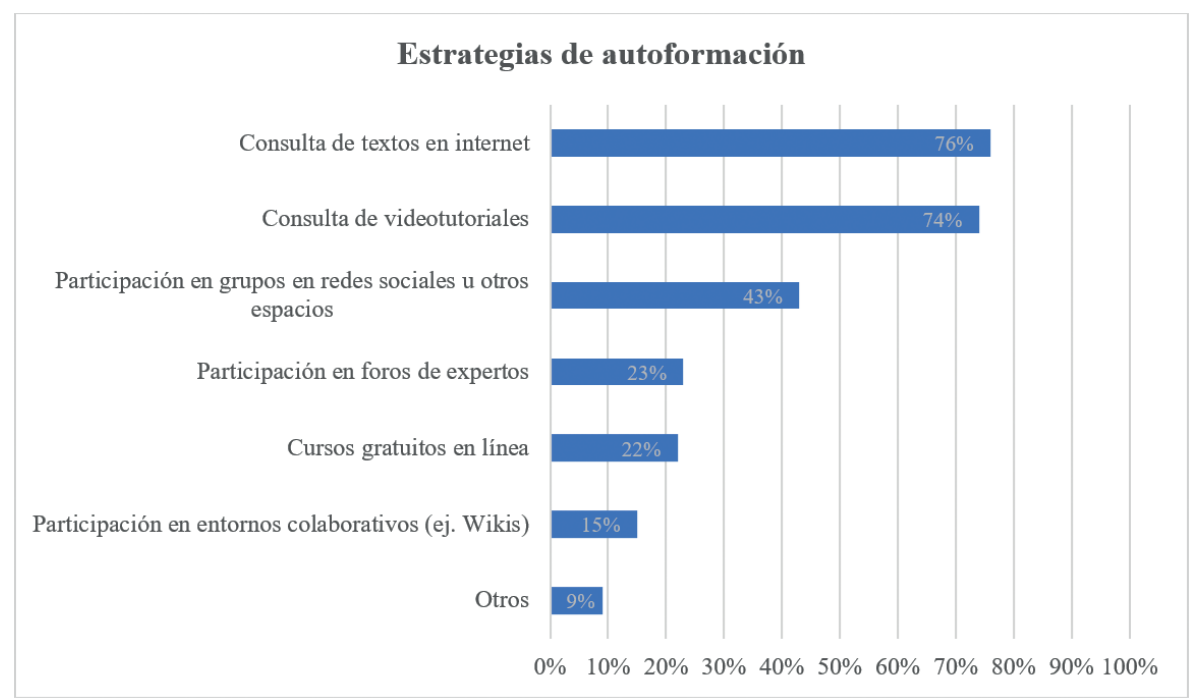

Figura 8. Estrategias de autoformación.

En una pregunta abierta del cuestionario se indagó por situaciones particulares en las que los estudiantes podían ejemplificar el uso de las estrategias de autoformación. Se destaca el uso de videotutoriales para resolver problemas técnicos con software básico o especializado (des- 
cargar o instalar un programa, resolver problemas con la impresora o cámara web). Entre los foros se mencionan Taringa y Yahoo! respuestas para resolver dudas específicas sobre el funcionamiento de las tecnologías. En menor medida, los estudiantes reconocen algunas plataformas gratuitas en las que pueden acceder a contenidos especializados a través de cursos masivos abiertos, entre estas Coursera y Actívate de Google.

Por otra parte, en los grupos de discusión se destacan las ventajas de llevar a cabo procesos de autoformación con recursos en línea, debido a que estos últimos suelen presentarse en un lenguaje más sencillo y cercano a las experiencias de los estudiantes, en modalidades de representación de la información más atractivas (visual y audiovisual) y con la posibilidad de ser consultados en distintas ocasiones y al propio ritmo, elementos que no siempre están presentes en el ámbito académico.

E5: "Muchas personas en Internet explican de manera clara ciertos conceptos o procesos y se pueden revisar las veces que uno quiera, no como en clase que un profesor te explica una vez".

E17: "Soy muy visual y prefiero aprender con imágenes o con animaciones, se me hace más fácil. Es más sencillo buscar algo en internet, más preciso, que un libro que tiene muchos rodeos de información".

\subsection{Herramientas y recursos digitales para el aprendizaje autónomo}

Esta categoría se refiere a las herramientas y recursos digitales que median el aprendizaje autónomo de los estudiantes.

En primer lugar, se indagó por herramientas generales en la web, entre las que se destaca el uso del buscador/navegador (96\%) como una metaherramienta que permite el acceso a otros recursos y que aporta a la resolución de problemas tecnológicos:

E2: "El navegador me parece a mí que es el más necesario. Si usted está leyendo algo y no entiende, si necesita buscar una palabra, un sinónimo, cómo se escribe o qué quiere decir, o no ha podido resolver un problema y va a Yahoo! respuestas, o necesita traducir, creo que todo se incluye allí mismo en el navegador".

Aparecen en menor medidas los traductores (59\%), las aplicaciones en los dispositivos móviles (55\%) y los diccionarios (15\%). 
En segundo lugar, se indagó por el uso de aplicaciones para el fortalecimiento de las competencias digitales. Al respecto, el $41 \%$ de los participantes indicó que hace uso de apps con propósitos de aprendizaje, pero es más frecuente que se usen con propósitos de entretenimiento (juegos) y en interacciones no formales (redes sociales, mensajería).

La figura 9 evidencia que los principales usos de las aplicaciones se relacionan con la interacción a través del correo o de redes sociales. También aparece con frecuencia el almacenamiento de archivos en la nube.

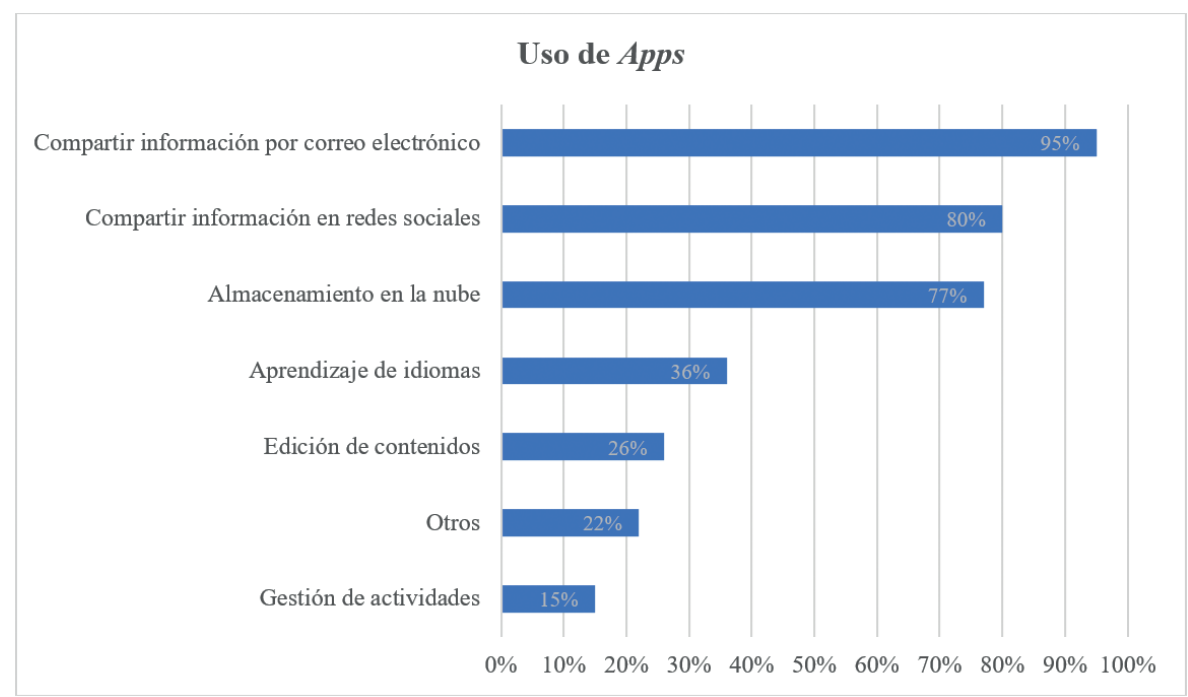

Figura 9. Uso de Apps.

\subsection{Posibilidades de transferencia al contexto académico}

Finalmente, se indagó con los estudiantes por las posibilidades de transferencia al contexto académico de sus habilidades digitales desarrolladas de forma autónoma en otros contextos. $46 \%$ de los estudiantes estuvieron de acuerdo en que es pertinente y necesaria esta articulación entre los saberes construidos en distintos ámbitos.

Entre las razones de aquellos que respondieron afirmativamente, se encuentra que las conexiones entre contextos formales e informales de aprendizaje pueden fortalecer la motivación de los estudiantes, al reconocer y validar otras capacidades que han construido por fuera de la escuela. También indican que sería favorable asumir características de 
estos aprendizajes, como actividades de interacción y colaboración con otros y la disminución de tensiones relacionadas con los procesos de evaluación.

E3: "Ayuda a aprovechar eso que ya tenemos y en lugar de prohibir las tecnologías se trata de integrarlas en lo que se hace en los salones de clase y con ayuda del profesor".

Por otra parte, los estudiantes que estuvieron en desacuerdo plantean que la universidad tiene unos propósitos de aprendizaje distintos a los que se dan en los contextos informales. De igual manera, indican que el contexto educativo formal es normativo, con poco espacio para la creatividad y la autonomía.

E2: "Las experiencias que tenido en las que usan las redes sociales para algún trabajo, por ejemplo, no son iguales que estar en una red social por voluntad propia. Se vuelve aburrido y con exceso de reglas".

\section{Discusión}

Los resultados de esta investigación evidencian que los participantes pasan gran parte de su tiempo en entornos digitales a través de sus dispositivos electrónicos fijos y móviles. De igual manera, los jóvenes han tenido experiencias que les han permitido desarrollar ciertas áreas de la competencia digital por fuera de la educación formal. No obstante, estas experiencias no son homogéneas, ni ubican necesariamente a los estudiantes como usuarios expertos en el manejo de todas las dimensiones de las tecnologías de la información y de la comunicación.

Entre las subcompetencias digitales percibidas con mayor desarrollo se sitúan la búsqueda de información, la interacción y el manejo de herramientas básicas. Estos resultados coinciden con los planteamientos de Castellanos, Sánchez y Calderero (2017) y Centeno y Cubo (2013), quienes afirman que las prácticas cotidianas de los millennials se centran en el uso de navegadores para satisfacer necesidades de información y en el uso de herramientas ofimáticas como el procesador de texto.

Tanto en la percepción del nivel de desempeño como en el inventario de aprendizajes digitales logrados de forma autogestionada, los estudian- 
tes destacan el manejo de las redes sociales. Dornaleteche et al., (2015) denominan este perfil de usuario como socializer, es decir, un perfil centrado en la interacción con propósitos sociales y recreativos. Gisbert y Esteve (2011) y Matamala (2016) coinciden con esta caracterización, al afirmar que la mayoría de los jóvenes desarrollan competencias digitales que les permiten participar en actividades de comunicación, entretenimiento y búsqueda de información.

El reconocimiento de las distintas dimensiones de la competencia digital, así como los niveles de desempeño de los grupos particulares de estudiantes con los que trabajamos, son de gran utilidad en la medida en que permiten identificar aquellas áreas que tienen un mayor desarroIlo y que pueden potenciarse en el ámbito académico (Gutiérrez et al., 2017). De igual forma, permiten evidenciar los aspectos que requieren de mayor atención. Particularmente, los resultados de esta investigación muestran necesidades en el establecimiento de criterios de evaluación de las fuentes de información (área 1), la participación ciudadana y la gestión de la imagen personal en entornos digitales (área 2), creación y modificación de software (área 3), el cuidado del entorno (área 4), la resolución de problemas con herramientas avanzadas, la innovación y el reconocimiento de vacíos en la competencia digital (área 5). Esta identificación ofrece a los docentes itinerarios de trabajo, en los que se trasciende la formación en aspectos técnicos básicos. Una mirada desde un enfoque holístico debe contemplar todas las dimensiones y ámbitos de la competencia digital y atender a cada aspecto de acuerdo con las características específicas de los estudiantes (Prendes, Martínez y Gutiérrez, 2017).

Un desafío importante respecto al desarrollo de las competencias digitales es que los aprendizajes construidos en otros contextos no siempre se transfieren al ámbito académico (Czerkawski 2016). Esto puede deberse a las representaciones que tanto estudiantes como docentes tienen sobre estas competencias, que pueden darse por sentadas ('invisibles' en términos de Cobo y Moravec, 2011) o considerarse poco relevantes. De hecho, en los resultados de este estudio se evidenció que la mitad de los jóvenes prefiere mantener la separación entre los aprendizajes de los contextos formales e informales.

Sin embargo, como plantea González (2009) "...si algo educa fuera del aula y sus procesos formales, es a nosotros [educadores] a quienes corresponde entenderlo" (p. 188). Es por esto que los profesores tenemos 
el compromiso de identificar cómo nuestros estudiantes construyen los conocimientos y desarrollan sus competencias y a partir de ello establecer posibles puentes con lo que sucede dentro del aula, asumiendo además que la educación actual supera los espacios físicos de la escuela y que debemos apuntar a una formación ubicua y que permita el aprendizaje a lo largo de la vida.

Una de las estrategias que puede aportar al establecimiento de estos puentes es reconocer las características de los aprendizajes que se dan por fuera de la escuela. En esta investigación los estudiantes indican que se autoforman principalmente a través de dos estrategias: consulta de información en la web y participación en comunidades en línea. Respecto a las competencias de alfabetización informacional, los profesores pueden aprovechar las habilidades de los estudiantes para localizar contenidos en distintas modalidades y plantear retos y necesidades de información relevantes. De igual manera, acompañar en aquellos aspectos que requieren mayor atención, como es el caso del establecimiento de criterios de análisis y evaluación de las fuentes, y potenciar el uso de recursos educativos digitales abiertos (Olcott, 2013).

Por otra parte, se puede atender a la creación de comunidades que compartan características con las comunidades de práctica o espacios de afinidad, en las que se construyen los aprendizajes de forma espontánea a partir de la interacción con los otros. Si bien la participación en grupos en línea no es la estrategia más usada por los estudiantes, es una de las que más fortalezas tiene, pues las interacciones pueden llevar a aprendizajes y formas de organización complejas y sistemáticas, como en el caso del Scanlation (Valero y Cassany, 2016). Una característica que llama la atención dentro de las comunidades de práctica es la forma de validar los conocimientos, ya que en estas no se llevan a cabo necesariamente procesos formales de evaluación. La validación de los saberes se puede dar de forma indirecta a través del reconocimiento social o el prestigio que puede alcanzar un usuario dentro de la comunidad, lo que aporta a procesos identitarios. También puede haber reconocimientos explícitos como las insignias digitales (Vázquez Cano et al., 2013), que podrían tener lugar en procesos de evaluación formal.

Debido a la alta frecuencia de uso de las redes sociales y a que los estudiantes construyen autónomamente las competencias digitales relacionadas con la comunicación y colaboración, una posible forma de establecer puentes entre los contextos es el uso de estas redes con fi- 
nes educativos (Lee y Sing, 2013). El reto en este caso es lograr que se mantenga la motivación y que las interacciones, si bien persiguen un propósito de aprendizaje, sean espontáneas y fluidas. Una ventaja de las redes sociales es que se puede hacer uso de las métricas que estas tienen incorporadas en su funcionamiento para evidenciar los impactos en los aprendizajes (Cuadros, 2015).

Como plantean Ricoy, Sevillano y Feliz (2011), “...para que la Sociedad del Conocimiento sea una realidad y continúe contribuyendo al progreso mundial es necesario que los ciudadanos puedan acceder a las TIC de modo normalizado en las respectivas facetas de la vida" (p. 504). De igual modo, desde los distintos contextos se deben hacer esfuerzos para establecer relaciones entre los aprendizajes y que se logren los propósitos de aprender a lo largo de la vida, aprovechando todas las experiencias, conocimientos y herramientas disponibles.

Los dispositivos móviles pueden jugar un papel fundamental en este propósito, debido a que los estudiantes los usan dentro y fuera de la escuela. Su uso con fines de aprendizaje podría optimizar las ventajas de la ubicuidad, aunque sin duda esto implica que las instituciones y el profesorado estén formados y se garanticen unas condiciones pedagógicas, didácticas y tecnológicas adecuadas (Vázquez Cano y Sevillano, 2017).

Finalmente, cabe considerar las limitaciones de un estudio de la competencia digital en contextos informales a través de instrumentos de autoobservación, debido a que pueden presentarse diferencias entre las percepciones de los estudiantes y las competencias que han desarrollado efectivamente. Por esta razón, se sugieren investigaciones que contrasten los discursos de los estudiantes con situaciones en las que se pongan en juego estas competencias.

\section{Referencias}

Ballesteros, B. y Mata, P. (2014). Investigación cualitativa en educación. Madrid: Editorial de la Universidad Nacional de Educación a Distancia.

Castellanos, A., Sánchez, C. y Calderero, J. F. (2017). Nuevos modelos tecnopedagógicos. Competencia digital de los alumnos universitarios. Revista Electrónica de Investigación Educativa, 19(1), 1-9. Recuperado de http://redie.uabc.mx/redie/article/ view/1148

Cassany, D. (2013). En línea: Leer y escribir en la red. España: Anagrama.

Centeno Moreno, G. y Cubo Delgado, S. (2013). Evaluación de la competencia digital y 
Desarrollo de competencias digitales de estudiantes universitarios en contextos informales de aprendizaje

Karen S. López-Gil y María luisa Sevillano García

las actitudes hacia las TIC del alumnado universitario. Revista de Investigación Educativa, 31 (2), 517-536. Recuperado de: http://dx.doi.org/10.6018/rie.31.2.169271

Cobo, C. y Moravec, J. (2011). Aprendizaje Invisible. Hacia una nueva ecología de la educación. Col-lecció Transmedia XXI. Laboratori de Mitjans Interactius / Barcelona: Publicacions i Edicions de la Universitat de Barcelona.

Comisión Europea. (2007). Competencias clave para el aprendizaje permanente un marco de referencia europeo. Luxemburgo: Oficina de Publicaciones Oficiales de las Comunidades Europeas.

Cross, J. (2006). Informal Learning: Rediscovering the Natural Pathways That Inspire Innovation and Performance. San Francisco: Jossey-Bass.

Cuadros, R. (2015). Aprendizaje informal y construcción de PLN vía twitter. Un estudio de caso. EDUTEC. Revista Electrónica de Tecnología Educativa, 51, 1-18.

Czerkawski, B. (2016). Blending Formal and Informal Learning Networks for Online Learning. International Review of Research un Open and Distributed Learning, 17(3), 138-156.

Domínguez, D. y Trillo Miravalles, P. (2014). Learning Competences in Open Mobile Environments: A Comparative Analysis Between Formal and Non-Formal Spaces. Open Praxis, 6(3), 235-244.

Dornaleteche-Ruiz, J., Buitrago-Alonso, A., y Moreno-Cardenal, L. (2015). Categorización, selección de ítems y aplicación del test de alfabetización digital online como indicador de la competencia mediática. Comunicar, XXII(44), 177-185.

Gee, J.P. (2017). Affinity Spaces and 21st Century Learning. Educational Technology, $57(2), 27-31$.

Gil Flores, J. (1992). La metodología de investigación mediante grupos de discusión. Enseñanza \& Teaching, (10-11), 199-214.

Gisbert, M. y Esteve, F. (2011). Digital Learners: la competencia digital de los estudiantes universitarios. La Cuestión Universitaria, 7, 48-59. Recuperado de: http://polired.upm. es/index.php/lacuestionuniversitaria/article/view/3359

González, R. (2009). La internet como espacio de producción de capital social: Una reflexión en torno a la idea de comunidad informal de aprendizaje. Revista Mexicana de Investigación Educativa, 14(40), 175-190.

Gutiérrez Castillo, J.J., Cabero Almenara, J. y Estrada-Vidal, L.I. (2017). Diseño y validación de un instrumento de evaluación de la competencia digital del estudiante universitario. Revista Espacios, 38(10).

Hernández, R., Fernández, C. y Baptista, P. (2014). Metodología de la investigación (6 ${ }^{\mathrm{a}}$ edición). México: Mc Graw Hill Education.

Infed. (2010). Informal Learning: theory, practice and experience. In: Infed: The informal education home page and encyclopaedia of informal education. Londres: YMCA George Williams College. Retrieved from: infed.org/mobi/informal-learning-theorypractice-and-experience/

Instituto Nacional de Tecnologías Educativas y de Formación del Profesorado - INTEF. (2017a). Resumen Informe Horizon. Edición 2017- Educación Superior. España: Gobierno de España.

Instituto Nacional de Tecnologías Educativas y de Formación del Profesorado - INTEF. 
Desarrollo de competencias digitales de estudiantes universitarios en contextos informales de aprendizaje

Karen S. López-Gil y María luisa SeVillano García

(2017b). Marco Común de Competencia Digital Docente. España: Gobierno de España.

Lankshear, C. \& Knobel, M. (2011). Nuevos alfabetismos: Su práctica cotidiana y el aprendizaje en el aula (3ra edición). España: Ediciones Morata.

Lee, B. \& Sing, A. (2013). Social media as an informal learning platform: Case study on adult learning at SIM University, Singapur. Procedia - Social and Behavioral Sciences, $93,1158-1161$.

Matamala, C. (2016). Uso de las TIC en el hogar: entre el entretenimiento y el aprendizaje informal. Estudios Pedagógicos, XLII(3), 293-311.

Olcott, D. (2013). Nuevas líneas de aprendizaje: potenciar el uso de recursos educativos abiertos para reforzar la educación no formal. RUSC. Universities and Knowledge Society Journal, 10(1), 151-169.

Paradise, R. (2005). Motivación e iniciativa en el aprendizaje informal. Sinéctica, Revista Electrónica de Educación, (26), 12-21.

Parlamento Europeo y Consejo de la Unión Europea. (2006). Recomendación del Parlamento Europeo y del Consejo de la Unión Europea sobre las competencias clave para el aprendizaje permanente. Diario Oficial de la Unión Europea.

Prendes Espinosa, M.P., Martínez Sánchez, F. y Gutiérrez Porlán, I. (2017). Competencia digital: una necesidad del profesorado universitario en el siglo XXI. RED Revista de Educación a Distancia, 56. Recuperado de: http://www.um.es/ead/red/56/prendes_et_al.pdf

Ricoy, M., Sevillano, M.L. y Feliz, T. (2011). Competencias necesarias para la utilización de las principales herramientas de Internet en la educación. Revista de Educación, 356, 483-507.

Sackey, S. Nguyen, M. \& Grabill, J. (2015). Constructing learning spaces: What we can learn from studies of informal learning online. Computers and Composition, 35, 112 124.

Unesco. (2010). Belém Framework for Action: Harnessing the power and potential of adult learning and education for a viable future. Hamburg, Germany: CONFITEA VI.

Unesco. (2016). Educación para la Ciudadanía Mundial. Preparar a los educandos para los retos del siglo XXI. París. Recuperado de http://unesdoc.unesco.org/ images/0024/002449/244957s.pdf

Valero, M. y Cassany, D. (2016). Traducción por fans para fans: organización y prácticas en una comunidad hispana de scanlation. BiD: textos universitaris de biblioteconomia i documentació, 37. Recuperado de: http://bid.ub.edu/es/37/cassany.htm.

Vázquez-Cano, E., López Meneses, E. y Sánchez-Serrano, L. (2013). La expansión del conocimiento en abierto: MOOC. Barcelona: Octaedro.

Vázquez-Cano, E. y Sevillano García, M.L. (2017). Lugares y espacios para el uso educativo y ubicuo de los dispositivos digitales móviles en la educación superior. Edutec. Revista Electrónica de Tecnología Educativa, 62, 48-61.

Wenger, E. (2000). Communities of Practice and Social Learning Systems. Electronic Article, 7(2) 225-246. Retrieved from: http://org.sagepub.com/ cgi/content/short/7/2/225 